\title{
Real-World Adherence and Persistence to Oral Disease-Modifying Therapies in Multiple Sclerosis Patients Over 1 Year
}

\author{
Kristen M. Johnson, PhD, MPH; Huanxue Zhou, MS; Feng Lin, PhD, MS; \\ John J. Ko, PharmD, MS; and Vivian Herrera, DDS, MIA, MPH
}

\begin{abstract}
BACKGROUND: Disease-modifying therapies (DMTs) are indicated to reduce relapse rates and slow disease progression for relapsing-remitting multiple sclerosis (MS) patients when taken as prescribed. Nonadherence or nonpersistence in the real-world setting can lead to greater risk for negative clinical outcomes. Although previous research has demonstrated greater adherence and persistence to oral DMTs compared with injectable DMTs, comparisons among oral DMTs are lacking.
\end{abstract}

OBJECTIVE: To compare adherence, persistence, and time to discontinuation among MS patients newly prescribed the oral DMTs fingolimod, dimethyl fumarate, or teriflunomide.

METHODS: This retrospective study used MarketScan Commercial and Medicare Supplemental claims databases. MS patients with $\geq 1$ claim for specified DMTs from April 1, 2013, to June 30, 2013, were identified. The index drug was defined as the first oral DMT within this period. To capture patients newly initiating index DMTs, patients could not have a claim for their index drugs in the previous 12 months. Baseline characteristics were described for patients in each treatment cohort. Adherence, as measured by medication possession ratio (MPR) and proportion of days covered (PDC); persistence (30-day gap allowed); and time to discontinuation over a 12-month follow-up period were compared across treatment cohorts. Adjusted logistic regression models were used to examine adherence, and Cox regression models estimated risk of discontinuation.

RESULTS: 1,498 patients newly initiated oral DMTs and met study inclusion criteria: fingolimod $(n=185)$, dimethyl fumarate $(n=1,160)$, and teriflunomide $(n=143)$. Patients were similar across most baseline characteristics, including region, relapse history, and health care resource utilization. Statistically significant differences were observed across the treatment cohorts for age, gender, previous injectable/infused DMT use, and comorbidities. Adherence and time to discontinuation were adjusted for age, gender, region, previous oral and injectable/infused DMT use, relapse history, and Charlson Comorbidity Index score. Relative to fingolimod patients, dimethyl fumarate and teriflunomide patients were significantly less likely to have an $\mathrm{MPR} \geq 80 \%(\mathrm{OR}=0.18 ; 95 \% \mathrm{Cl}=0.09-0.36 ; P<0.001$ and $\mathrm{OR}=0.19$; $95 \% \mathrm{Cl}=0.08-0.42 ; P<0.001$, respectively). Similarly, relative to fingolimod patients, dimethyl fumarate and teriflunomide patients were significantly less likely to have $\mathrm{PDC} \geq 80 \%(0 \mathrm{R}=0.47 ; 95 \% \mathrm{Cl}=0.33-0.67 ; P<0.001$ and $\mathrm{OR}=0.37 ; 95 \% \mathrm{Cl}=0.23-0.59 ; P<0.001$, respectively). Additionally, the HR for discontinuation was about 2 times greater for dimethyl fumarate $(\mathrm{HR}=1.93 ; 95 \% \mathrm{Cl}=1.44-2.59 ; P<0.001)$ and teriflunomide patients ( $\mathrm{HR}=2.27 ; 95 \% \mathrm{Cl}=1.57-3.28 ; P<0.001)$ compared with fingolimod.

CONCLUSIONS: In a real-world setting, patients taking fingolimod had better adherence and persistence compared with patients taking other oral DMTs over 12 months. Coupled with clinical factors, medication adherence and persistence should be important considerations when determining coverage decisions for MS patients.

J Manag Care Spec Pharm. 2017;23(8):844-52

Copyright $\odot 2017$, Academy of Managed Care Pharmacy. All rights reserved.

\section{What is already known about this subject}

Disease-modifying therapies (DMTs) are the cornerstone of treatment for relapsing forms of multiple sclerosis (MS), with therapeutic goals being to reduce the frequency and severity of relapses, reduce central nervous system lesions, and slow the accumulation of disability.

Poor adherence and persistence to medications are common across many conditions, including MS, and real-world studies have shown that not taking DMTs as prescribed can lead to greater risk for negative clinical outcomes.

Previous research has demonstrated greater adherence and persistence to oral DMTs compared with injectable DMTs; however, more real-world comparisons are needed among newer DMTs.

\section{What this study adds}

This study is the first to compare adherence and persistence among patients initiating the oral DMTs fingolimod, dimethyl fumarate, and teriflunomide over 1 year in a real-world setting. When adjusted for baseline characteristics, relative to fingolimod patients, dimethyl fumarate and teriflunomide patients were significantly less likely to have a medication possession ratio $\geq 80 \%$, and a similar result was observed when using proportion of days covered to measure adherence.

When adjusted for baseline characteristics, the risk of treatment discontinuation was almost 2 times greater for dimethyl fumarate patients compared with fingolimod patients, and teriflunomide patients had more than 2 times increased risk for discontinuation compared with fingolimod patients.

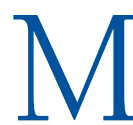
ultiple sclerosis (MS) is a heterogeneous, unpredictable, and often disabling disease caused by damage to the central nervous system, including the brain, spinal cord, or optic nerves. ${ }^{1}$ As a generally progressive disease, it is associated with significant humanistic and economic burden. ${ }^{2}$ Approximately $85 \%$ of individuals with MS have an initial disease course that is relapsing and remitting in nature (i.e., relapsing-remitting multiple sclerosis [RRMS]), characterized by neurological attacks followed by periods of complete or partial recovery and absence of clinical disease progression. Over time, most individuals with RRMS transition to a more progressive form of the disease, characterized by steady 
disease progression without remission. ${ }^{1}$ It is estimated that MS affects approximately 2.5 million people globally, with 400,000 people in the United States. ${ }^{3}$ MS typically becomes clinically apparent between the ages of 15 and 45 years and is 2 to 3 times more common in women than in men. ${ }^{4}$

Disease-modifying therapies (DMTs) are the cornerstone of treatment for RRMS, with therapeutic goals being to reduce the frequency and severity of relapses, reduce central nervous system lesions, and slow the accumulation of disability. ${ }^{5}$ There are multiple DMTs available for the treatment of relapsing forms of MS. Before 2010, only DMTs administered by injection or intravenous infusion were available. In September 2010, fingolimod was the first oral DMT approved by the U.S. Food and Drug Administration for relapsing forms of MS. ${ }^{6}$ Since then, 2 oral DMTs with different mechanisms of action have been approved in the United States: teriflunomide in September 2012 and dimethyl fumarate in March 2013.7,8 The Multiple Sclerosis Coalition consensus paper on the use of DMTs in MS does not make specific comparisons or recommendations on which DMTs should be used. ${ }^{9}$ Rather, because available treatments have demonstrated efficacy in relapsing MS and factors that influence choice of medication are complex, the use of a specific DMT should be made collaboratively by patients and their treating clinicians.

For medications to be most effective, patients must take them as prescribed by their health care providers (i.e., be adherent) and for the prescribed duration of treatment (i.e., be persistent). ${ }^{10,11}$ However, poor adherence and nonpersistence are common across many conditions, including MS. For example, a review of 24 adherence studies of injectable DMTs found that adherence ranged from $41 \%$ to $88 \% .{ }^{12}$ A retrospective claims database study of MS patients initiating therapy with 1 of 4 injectable DMTs found that after 18 months, $11.2 \%$ of patients switched their medication, and 33.9\% discontinued their index therapy, although switch and discontinuation rates differed by index DMT used. ${ }^{13}$

Poor adherence and nonpersistence occur for a variety of reasons, which can be attributable to patients, physicians, treatment regimens, and health care systems. ${ }^{10}$ Previous research has found that nonadherence and nonpersistence among MS patients using DMTs can be due to disease factors (e.g., continued relapses and disease progression), cognitive impairment and depression, lack of treatment efficacy, drug safety or tolerability concerns, treatment inconvenience, injection burden of intravenous and injectable DMTs, and costs to patients for DMT treatment. ${ }^{14-16}$ A recent survey of MS patients who were treatment naive to oral DMTs examined potential reasons for nonadherence to a hypothetical oral DMT and found that the most important determinants of potential nonadherence were the frequency of daily administration, the safety of DMTs during pregnancy, and side effects, including hair thinning. ${ }^{17}$ These findings suggest that determinants of nonadherence or nonpersistence may differ among injectable and oral DMTs.
Real-world studies have shown that not taking DMTs as prescribed (i.e., nonadherence or nonpersistence) can lead to greater risk for negative clinical and economic outcomes. ${ }^{18,19}$ In a retrospective claims database study, Ivanova et al. (2012) compared rates of severe MS relapse and total direct and indirect costs between U.S. patients with MS who were adherent and nonadherent to injectable or intravenous DMTs. ${ }^{18}$ Over the 24-month study period, $69.1 \%$ of patients were classified as adherent using the commonly accepted threshold of medication possession ratio (MPR) $\geq 80 \%$. After controlling for baseline differences, the risk-adjusted rate of severe relapse during the study period was significantly lower for adherent patients than nonadherent patients (12.4\% vs. 19.9\%). In addition, the total risk-adjusted costs (excluding DMT costs) were significantly lower among adherent patients than nonadherent patients $(\$ 14,095$ vs. $\$ 16,638) .^{18}$

In another retrospective database study, Tan et al. (2011) assessed the impact of adherence to injectable DMTs on MS-related health care resource utilization, MS relapses, and medical costs among commercially insured MS patients in the United States. ${ }^{19}$ Among 2,446 MS patients, 59.6\% were adherent to their DMTs during the 12-month study follow-up period (defined as MPR $\geq 80 \%$ ). Adjusted results showed that adherent patients were significantly less likely to have an MS-related hospitalization and to have an MS relapse than nonadherent patients. Regarding MS-related medical costs, adjusted results showed that the adherent group on average had 22\% lower MS-related medical costs than the nonadherent group during the study period. ${ }^{19}$

Previous research has demonstrated greater adherence and persistence to oral DMTs compared with injectable DMTs ${ }^{20,21}$; however, real-world comparisons among all approved oral DMTs are lacking. The objective of this study was to compare adherence, persistence, and time to discontinuation among MS patients in U.S. commercial and Medicare supplemental health plans who newly initiated the oral DMTs fingolimod, dimethyl fumarate, or teriflunomide.

\section{Methods}

\section{Study Design and Data Sources}

This retrospective claims database study used the MarketScan Commercial and Medicare Supplemental administrative claims databases. MarketScan offers the largest convenience sample (over 230 million de-identified patients) available in proprietary databases, which is sourced from large employers, managed care organizations, hospitals, and Medicare and Medicaid programs. The databases reflect the full continuum of care across all health care providers and settings. De-identified claims information includes inpatient admission records, outpatient services, prescription drugs, eligibility status, and costs of services. The medical and pharmacy claims are linked to person-level enrollment information. All MarketScan data 


\section{TABLE 1 Patient Selection}

\begin{tabular}{|c|c|c|c|}
\hline \multirow{3}{*}{$\begin{array}{l}\text { Inclusion and Exclusion Criteria } \\
\text { Patients with multiple sclerosisa and with at least } 1 \text { claim for fingolimod, dimethyl } \\
\text { fumarate, or teriflunomide from April 1, 2013, to June 30, } 2013\end{array}$} & \multicolumn{3}{|c|}{ Sample Size } \\
\hline & \multicolumn{3}{|c|}{6,243} \\
\hline & Fingolimod & Dimethyl Fumarate & Teriflunomide \\
\hline Patients at index treatment ${ }^{\mathrm{b}}$ & 2,968 & 2,602 & 673 \\
\hline Aged 18 years or older at index date ${ }^{c}$ & 2,968 & 2,600 & 673 \\
\hline $\begin{array}{l}\text { Patients continuously enrolled in medical and pharmacy benefits from index date to } \\
12 \text {-month follow-up }\end{array}$ & 2,380 & 1,931 & 505 \\
\hline Patients continuously enrolled in medical and pharmacy benefits in 365 days before index date & 1,914 & 1,514 & 401 \\
\hline Excluded patients with $\geq 20$ claims for 1 NDC number in 12 -month follow-up period & 1,913 & 1,510 & 399 \\
\hline Excluded patients with any claims for index treatment in 12 months before index date & 196 & 1,510 & 144 \\
\hline Excluded patients in multiple cohorts & 195 & 1,509 & 143 \\
\hline $\begin{array}{l}\text { For dimethyl fumarate cohort only: excluded patients with only l-day starter pack in the } \\
\text { entire study period }\end{array}$ & & 1,508 & \\
\hline $\begin{array}{l}\text { For dimethyl fumarate cohort only: included patients who initiated dimethyl fumarate } \\
\text { starter pack only }\end{array}$ & & 1,160 & \\
\hline \multicolumn{4}{|c|}{$\begin{array}{l}\text { aAt least } 1 \text { claim with an ICD-9-CM diagnosis code of multiple sclerosis (340). } \\
\text { bThe date of first medication claim is the index date. } \\
\text { cThe index drug was defined as the first newly initiated oral DMT (new starts) within the identification period. } \\
\text { DMT = disease-modifying therapy; ICD-9-CM = International Classification of Diseases, Ninth Revision, Clinical Modification; NDC=National Drug Code. }\end{array}$} \\
\hline
\end{tabular}

are fully compliant with the Health Insurance Portability and Accountability Act.

\section{Patient Population}

MS patients (International Classification of Diseases, Ninth Revision, Clinical Modification [ICD-9-CM] code 340) with at least 1 pharmacy claim for fingolimod, dimethyl fumarate, or teriflunomide between April 1, 2013, and June 30, 2013, (identification period) were identified in the databases using National Drug Code (NDC) numbers. The date of the first claim for fingolimod, dimethyl fumarate, or teriflunomide within the identification period was defined as the index date. The index drug was defined as the first oral DMT within the identification period. Patients were assigned to a treatment cohort based on their index drugs.

Study inclusion and exclusion criteria and associated patient flow are described in Table 1. Patients were included in the study sample if they were aged 18 years or older; had at least 1 claim for fingolimod, dimethyl fumarate, or teriflunomide in the identification period; and were continuously enrolled in medical and pharmacy benefits for 12 months before the index date and for the entire 12-month follow-up period. Patients who had claims for their index drugs in the 12-month pre-index period were excluded to ensure that all patients had newly initiated each of the index oral DMTs. Patients in each group were allowed to have previously used 1 of the other oral DMTs before the index date. Patients were also excluded if they had $\geq 20$ claims for any single NDC number in the 12-month follow-up period or had claims for multiple index drugs during the follow-up period. For the dimethyl fumarate cohort, patients were included if they initiated on the dimethyl fumarate starter pack (comprising $120 \mathrm{mg}$ for the first 7 days followed by $240 \mathrm{mg}$ ) within the identification period.

Patients were followed for up to 12 months after their index dates to evaluate adherence and persistence of index drugs at recommended doses: fingolimod $0.5 \mathrm{mg}$ daily; dimethyl fumarate $120 \mathrm{mg}$ daily for 7 days followed by $240 \mathrm{mg}$ daily for 21 days (i.e., starter pack regimen); and teriflunomide $7 \mathrm{mg}$ or 14 mg daily.

\section{Study Measures}

Baseline Characteristics. The study cohorts were described using baseline demographic and clinical characteristics and health care resource utilization. Demographic characteristics included age, gender, insurance plan type, and geographic region. Baseline clinical characteristics included previous DMT use, the number of MS relapses, all-cause and MS-related health care resource utilization, and comorbidity indices during the 12 months before the index date. An MS relapse was defined as a claim with an MS diagnosis code (ICD-9-CM code 340) in the primary position at any time during an inpatient hospitalization or a claim with an MS diagnosis code in the primary or secondary position in an outpatient setting (emergency room [ER] and physician office visit) in addition to a pharmacy or medical claim for a qualifying corticosteroid on the day of or within 7 days after a visit. A "clean period" was defined where relapses had to be at least 30 days apart to be counted as more than 1 relapse (i.e., if more than 1 relapse occurred within a 30-day period, it was only counted as a single relapse event). ${ }^{22}$ 
TABLE 2 Baseline Demographic and Clinical Characteristics ${ }^{a}$

\begin{tabular}{|c|c|c|c|c|c|c|c|}
\hline \multirow[b]{2}{*}{ Patients, n (\%) } & \multicolumn{2}{|c|}{ Fingolimod } & \multicolumn{2}{|c|}{ Dimethyl Fumarate } & \multicolumn{2}{|c|}{ Teriflunomide } & \multirow[t]{2}{*}{$P$ Value $^{\text {b }}$} \\
\hline & 195 & 13.0 & 1,160 & 77.4 & 143 & 9.5 & \\
\hline Age, mean (SD) & 44.4 & 10.6 & 48.1 & 10.6 & 53.2 & 9.9 & $<0.001$ \\
\hline Gender, n (\%) & & & & & & & 0.048 \\
\hline Male & 32 & 16.4 & 284 & 24.5 & 33 & 23.1 & \\
\hline Female & 163 & 83.6 & 876 & 75.5 & 110 & 76.9 & \\
\hline Plan type, n (\%) & & & & & & & 0.254 \\
\hline Fee-for-service & 160 & 82.1 & 978 & 84.3 & 127 & 88.8 & \\
\hline HMO and POS capitation & 20 & 10.3 & 118 & 10.2 & 13 & 9.1 & \\
\hline Unknown & 15 & 7.7 & 64 & 5.5 & 3 & 2.1 & \\
\hline Region, n (\%) & & & & & & & 0.477 \\
\hline Northeast & 38 & 19.5 & 273 & 23.5 & 26 & 18.2 & \\
\hline North Central & 50 & 25.6 & 262 & 22.6 & 40 & 28.0 & \\
\hline South & 62 & 31.8 & 324 & 27.9 & 41 & 28.7 & \\
\hline West & 41 & 21.0 & 274 & 23.6 & 35 & 24.5 & \\
\hline Unknown & 4 & 2.1 & 27 & 2.3 & 1 & 0.7 & \\
\hline Previous injectable/infused DMT use, n (\%) & 130 & 66.7 & 838 & 72.2 & 91 & 63.6 & 0.043 \\
\hline Previous other oral DMT use, n (\%) & 1 & 0.5 & 39 & 3.4 & 6 & 4.2 & 0.073 \\
\hline MS relapse, mean (SD) & 0.33 & 0.61 & 0.37 & 0.72 & 0.50 & 0.86 & 0.198 \\
\hline$\geq 1$ MS relapse, $\mathrm{n}(\%)$ & 53 & 27.2 & 310 & 26.7 & 47 & 32.9 & 0.298 \\
\hline \multicolumn{8}{|l|}{ Resource utilization, n (\%) } \\
\hline All-cause hospitalization & 23 & 11.8 & 128 & 11.0 & 18 & 12.6 & 0.833 \\
\hline All-cause ER visit & 57 & 29.2 & 307 & 26.5 & 34 & 23.8 & 0.523 \\
\hline All-cause office visit & 194 & 99.5 & 1,155 & 99.6 & 142 & 99.3 & 1.000 \\
\hline MS-related hospitalization & 10 & 5.1 & 43 & 3.7 & 9 & 6.3 & 0.259 \\
\hline MS-related ER visit & 26 & 13.3 & 152 & 13.1 & 23 & 16.1 & 0.614 \\
\hline MS-related office visit & 191 & 97.9 & 1,134 & 97.8 & 141 & 98.6 & 0.823 \\
\hline Charlson Comorbidity Index score, mean (SD) & 0.33 & 0.86 & 0.44 & 0.90 & 0.68 & 1.28 & 0.003 \\
\hline Elixhauser Comorbidity Index score, mean (SD) & 2.11 & 1.47 & 2.08 & 1.28 & 2.42 & 1.97 & 0.579 \\
\hline \multicolumn{8}{|c|}{$\begin{array}{l}\text { aThe demographic information was measured at index date. Disease history, including previous DMT use and resource utilization and comorbidity information, was } \\
\text { measured } 12 \text { months before index date. } \\
\text { bChi-square tests (Fisher exact test was employed when at least } 20 \% \text { of the cells have an expected value less than 5) for categorical/dummy variables and Kruskal-Wallis } \\
\text { test for continuous variables were performed. } \\
D M T \text { disease-modifying therapy; ER=emergency room; HMO=health maintenance organization; MS = multiple sclerosis; POS=point of service; SD=standard deviation }\end{array}$} \\
\hline
\end{tabular}

Health care resource utilization included all-cause hospitalizations, all-cause ER visits (inpatient and outpatient), and all-cause office visits. MS-related hospitalizations (MS diagnosis code in the primary position); MS-related ER visits (MS diagnosis code in the primary position for ER in the inpatient setting and MS diagnosis code in the primary or secondary position for ER in the outpatient setting); and MS-related office visits (MS as the primary or secondary diagnosis) were also analyzed.

Comorbidity indices included the Charlson Comorbidity Index (CCI) score and the Elixhauser Comorbidity Index score for use with administrative claims data. ${ }^{23-25}$ The CCI is an aggregate measure of comorbidity created using select diagnoses associated with chronic diseases, and the Elixhauser Comorbidity Index measures 31 comorbid conditions using ICD-9-CM codes and is meant to be used with large administrative datasets. ${ }^{25}$
Outcome Measures. All outcomes were assessed for the 12 months following the index date. Outcome measures included treatment adherence, treatment persistence, and risk of treatment discontinuation. Adherence was measured by pharmacy claims using MPR and proportion of days covered (PDC), which are commonly used measures for assessing treatment adherence in administrative claims studies. ${ }^{26}$ For each treatment cohort, adherence measured with MPR was assessed in the subset of patients who had at least 2 claims for the index DMT during the 12-month follow-up period. MPR was estimated as the total days with index medication supply within the refill interval divided by the number of days between the first prescription date and the last prescription date. PDC was estimated as the number of days with index medication supply divided by the number fixed days in the follow-up period (i.e., 365 days). Using a commonly accepted threshold, ${ }^{26}$ patients with MPRs or PDCs $\geq 80 \%$ were considered adherent to their 
index DMTs, whereas patients with MPRs or PDCs $<80 \%$ were considered nonadherent. Treatment persistence for the index DMT was defined as the proportion of patients who remained on their index DMTs with less than a 30-day gap during the 12-month follow-up period.

\section{Statistical Analyses}

Descriptive analyses for demographics, baseline characteristics, and outcomes were conducted and reported for each treatment cohort. For categorical variables, counts and percentages are provided. Means and standard deviations (SDs) are presented for continuous variables. To compare outcomes of the cohorts, chi-square tests (Fisher exact test was used when $\geq 20 \%$ of the cells had an expected value less than 5) were performed for categorical variables, and Kruskal-Wallis tests were used for continuous variables. Multivariable logistic regression models adjusting for differences among the treatment cohorts were used to assess adherence. Time to discontinuation from the index drug was described using Kaplan-Meier analysis. A Cox proportional hazards model adjusting for differences among the treatment cohorts was used to assess the risk of discontinuation. Covariates in the adjusted analyses included age, gender, region, previous oral and injectable/infused DMT use (yes/no), MS relapse during the baseline period (yes/no), and CCI score. To evaluate sensitivity of the 30-day grace period in the risk of discontinuation assessment, a sensitivity analysis was conducted using a 60-day grace period.

\section{Results}

\section{Baseline Characteristics}

Of the 1,498 patients who newly initiated oral DMTs and met study inclusion and exclusion criteria, $13.0 \%$ initiated fingolimod, $77.4 \%$ dimethyl fumarate, and $9.5 \%$ teriflunomide. Patient baseline demographic characteristics, clinical characteristics, and health care resource utilization are shown in Table 2. In general, patients were similar across baseline demographic characteristics, including region, relapse history, and health care resource utilization. Statistically significant differences were observed across the treatment cohorts for age, gender, previous injectable/infused DMT use, and comorbidities. The mean age for the treatment cohorts ranged from 44.4 to 53.2 years, with patients in the teriflunomide group having the highest mean age. The majority of patients were female (75.5\%-83.6\%). About two thirds of the patients had previous injectable/infused DMT use during the baseline period, whereas fewer than $5 \%$ had previous oral DMT use. Patients in the teriflunomide group also had higher scores on the comorbidity indices.

\section{Medication Adherence}

Fingolimod patients had higher unadjusted MPR (mean $=93.2 \%$ $[S D=9.0]$ ) than dimethyl fumarate patients (mean $=87.8 \%$

\begin{tabular}{|c|c|c|}
\hline \multicolumn{3}{|c|}{$\begin{array}{l}\text { Adjusted Adherence Outcomes } \\
\text { for Oral DMT Cohorts }\end{array}$} \\
\hline Parameter & OR $(95 \% \mathrm{CI})$ & $P$ Value \\
\hline \multicolumn{3}{|l|}{ MPR $\geq 80 \%$} \\
\hline Fingolimod & Reference & \\
\hline Dimethyl fumarate & $0.18 \quad(0.09-0.36)$ & $<0.001$ \\
\hline Teriflunomide & $0.19 \quad(0.08-0.42)$ & $<0.001$ \\
\hline \multicolumn{3}{|l|}{ PDC $\geq 80 \%$} \\
\hline Fingolimod & Reference & \\
\hline Dimethyl fumarate & $0.47 \quad(0.33-0.67)$ & $<0.001$ \\
\hline Teriflunomide & $0.37 \quad(0.23-0.59)$ & $<0.001$ \\
\hline \multicolumn{3}{|c|}{$\begin{array}{l}\text { aAdherence outcomes were adjusted for region, gender, age, previous injectable/ } \\
\text { infused DMT use (yes/no), previous oral DMT use (yes/no), previous MS relapse } \\
\text { (yes/no), and CCI score. } \\
C C I=\text { Charlson Comorbidity Index; CI = confidence interval; DMT=disease- } \\
\text { modifying therapy; MPR=medication possession ratio; } M S=\text { multiple sclerosis; } \\
\text { OR=odds ratio; } P D C=\text { proportion of days covered. }\end{array}$} \\
\hline
\end{tabular}

$[S D=16.8]$ ) and teriflunomide patients (mean $=88.3 \%$ [SD = 16.1], $P=0.03$ ). Similarly, fingolimod patients had higher unadjusted PDC (mean $=81.4 \% \quad[S D=27.3])$ than dimethyl fumarate patients (mean $=71.0 \%[S D=32.7]$ ) and teriflunomide patients (mean $=68.2 \%[S D=32.0], P<0.001$ ). In unadjusted analyses, significantly more fingolimod patients were adherent at 12 months (88.7\%) compared with dimethyl fumarate (70.6\%) and teriflunomide $(72.7 \%)$ patients as measured by MPR $\geq 80 \%$ $(P<0.001)$. Results were consistent when using the more conservative calculation of $\mathrm{PDC} \geq 80 \%$ to measure adherence at 12 months: fingolimod (75.4\%), dimethyl fumarate (60.3\%), and teriflunomide $(53.1 \% ; P<0.001)$. The adjusted adherence analyses are presented in Table 3. Relative to fingolimod patients, dimethyl fumarate and teriflunomide patients were significantly less likely to have an $\mathrm{MPR} \geq 80 \%$ (odds ratio $[\mathrm{OR}]=0.18$ and $\mathrm{OR}=0.19$, respectively; both $P<0.001$ ). Similarly, dimethyl fumarate and teriflunomide patients were significantly less likely to have a $\mathrm{PDC} \geq 80 \%(\mathrm{OR}=0.47$ and $\mathrm{OR}=0.37$, respectively; both $P<0.001)$ compared with fingolimod.

\section{Persistence and Time to Discontinuation}

During the 12-month follow-up, a significantly higher percentage of fingolimod patients were persistent on therapy compared with dimethyl fumarate and teriflunomide patients $(74.4 \%$, $55.9 \%$, and $49.7 \%$, respectively; $P<0.001$ ). The mean time to discontinuation for fingolimod, dimethyl fumarate, and teriflunomide patients was 253, 211, and 202 days, respectively $(P<0.001)$. The unadjusted time to discontinuation by DMT cohort using Kaplan-Meier analysis is shown in Figure 1. The probability of patients being persistent on index DMTs over the study period (300 days of follow-up to allow discontinuation with a 60-day gap to be assessed within the 1-year period) was significantly higher for fingolimod patients compared with dimethyl fumarate and teriflunomide patients $(P<0.001)$. 


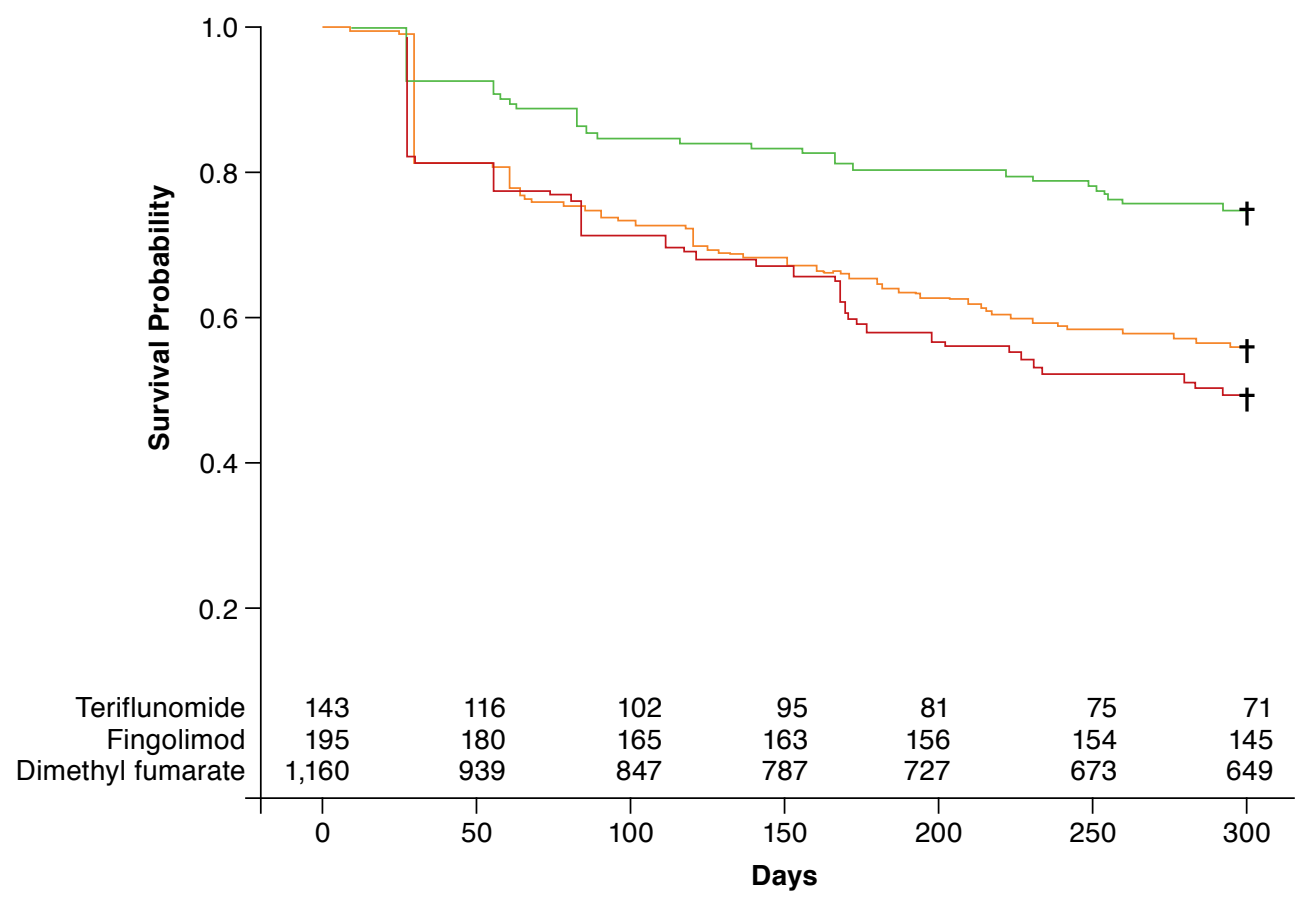

Cohort
†Censored

\section{Risk of Discontinuation}

When adjusted for baseline differences, the hazard of discontinuation with a 30-day grace period was almost 2 times greater for dimethyl fumarate patients (hazard ratio $[\mathrm{HR}]=1.93$; 95\% confidence interval $[\mathrm{CI}]=1.44-2.59 ; \mathrm{P}<0.001)$ compared with fingolimod patients (Figure 2). Similarly, teriflunomide patients had more than 2 times greater risk for discontinuation compared with fingolimod patients (HR $=2.27 ; 95 \% \mathrm{CI}=1.57$ 3.28; $P<0.001)$.

\section{Sensitivity Analysis Using a 60-Day Grace Period}

When a 60-day grace period was assessed as a sensitivity analysis, the results were similar to a 30 -day grace period. The proportion of patients remaining on fingolimod, dimethyl fumarate, and teriflunomide during the 12-month follow-up period was $79.0 \%, 65.6 \%$, and $58.7 \%$, respectively $(P<0.001)$. The mean time to treatment discontinuation for the fingolimod, dimethyl fumarate, and teriflunomide cohorts was 262.6, 231.1, and 227.7 days, respectively $(P<0.001)$. Adjusting for baseline characteristics, dimethyl fumarate $(H R=1.76 ; 95 \% C I=1.28-2.44 ; P<0.001)$ and teriflunomide patients $(\mathrm{HR}=2.06 ; \mathrm{CI}=1.37-3.09 ; P<0.001)$ had approximately 2 times greater hazard of discontinuation compared with fingolimod patients.

\section{Discussion}

This U.S. retrospective database study is the first to compare treatment adherence and persistence among patients who initiated the oral DMTs fingolimod, dimethyl fumarate, and teriflunomide over 1 year in a real-world setting. Compared with dimethyl fumarate and teriflunomide, fingolimod was associated with significantly greater adherence over 12 months, with $88.7 \%$ of patients considered adherent as measured by an MPR of $\geq 80 \%$ and $75.4 \%$ considered adherent as measured by PDC $\geq 80 \%$. Adjusted analyses showed that relative to fingolimod patients, dimethyl fumarate and teriflunomide patients were significantly less likely to have MPR and PDC $\geq 80 \%$. Also, a higher percentage of fingolimod patients (74.4\%) were persistent on therapy compared with dimethyl fumarate and teriflunomide patients (55.9\% and $49.7 \%$, respectively), and the mean time to discontinuation was longest for fingolimod 


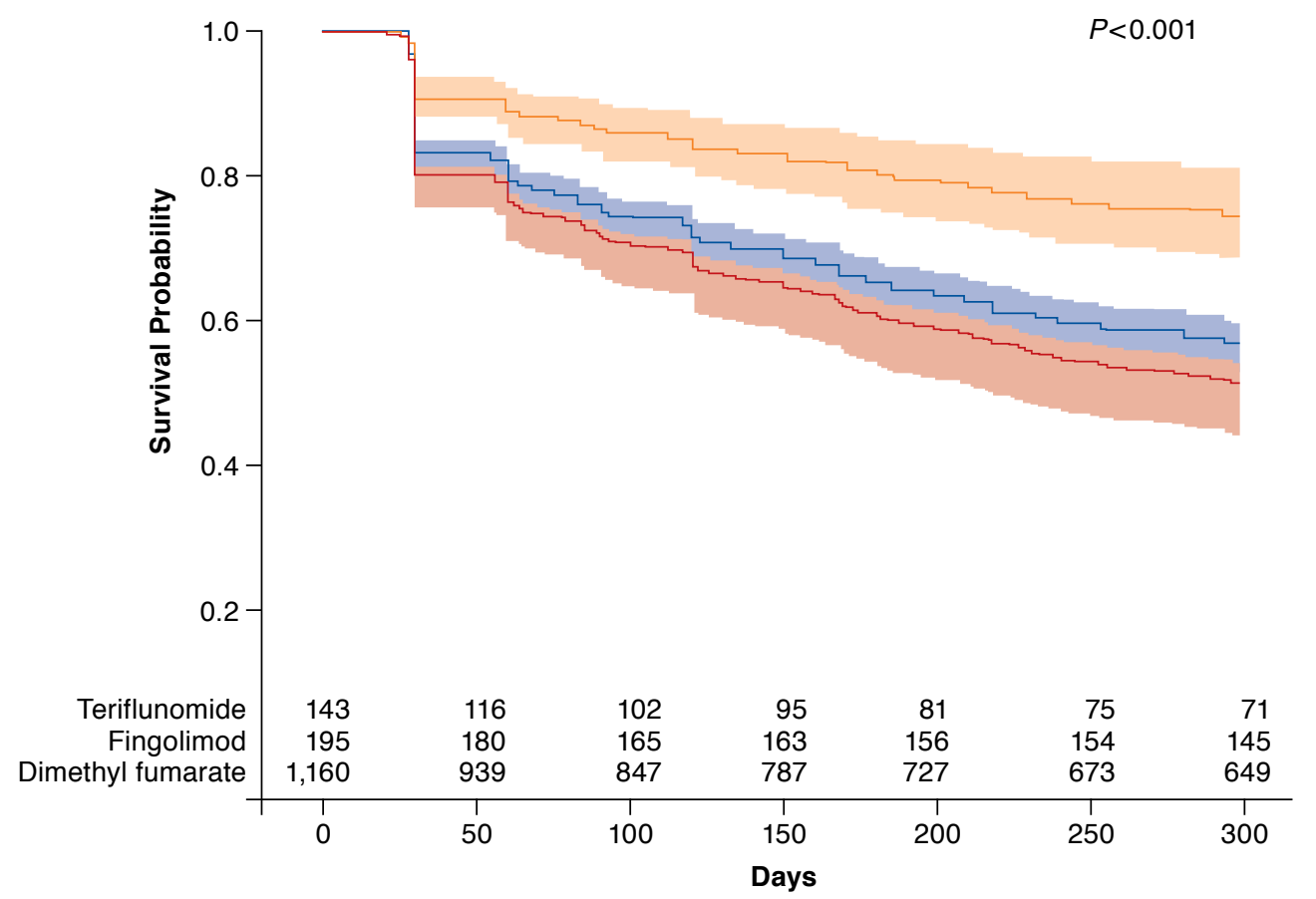

Cohort

Teriflunomide $=$ Fingolimod $=$ Dimyl fumarate

aAdherence outcomes were adjusted for region, gender, age, previous injectable/infused DMT use (yes/no), previous oral DMT use (yes/no), previous MS relapse (yes/no), and CCI score.

$C C I=$ Charlson Comorbidity Index; DMT = disease-modifying therapy; $M S=$ multiple sclerosis .

(252.9 days). Adjusted analyses found that the risk of discontinuation was about 2 times greater for teriflunomide and dimethyl fumarate compared with fingolimod.

Previous research has shown better treatment adherence and persistence among fingolimod patients compared with patients using injectable or intravenous DMTs. In a retrospective administrative claims database study, ${ }^{21}$ the proportion of patients who were persistent on their index therapy after 360 days was significantly higher in the fingolimod cohort (72.1\%) compared with the glatiramer acetate $(60.5 \%)$, interferon (56.3\%), and natalizumab (61\%) cohorts (all, $P<0.001$ ). Similar to the risk of discontinuation results seen in our study of oral DMTs, risk of discontinuation was 1.5- to 2-fold higher (all, $P<0.001)$ in patients taking injectable DMTs compared with patients taking fingolimod. In addition, fingolimod patients demonstrated high adherence and the lowest proportion of nonadherent patients compared with the other treatment cohorts as measured by MPR (6.2\% nonadherent) and PDC (10.3\% nonadherent). ${ }^{21}$
Another retrospective database study that examined adherence and persistence of fingolimod compared with injectable DMTs found similar results. ${ }^{20}$ In this study, patients who initiated fingolimod had the highest average PDC and MPR in experienced (fingolimod: $73.7 \%$ with $\mathrm{PDC} \geq 80 \%$; $90.5 \%$ with $\mathrm{MPR} \geq 80 \%$ ) and naive DMT users (fingolimod: $66.7 \%$ with $\mathrm{PDC} \geq 80 \% ; 87.4 \%$ with $\mathrm{MPR} \geq 80 \%$ ). The proportion of patients who discontinued their index DMTs within 12 months was significantly lower for the fingolimod cohort (naive: $31.3 \%$; experienced: $25.7 \%$ ). Adjusted results found that patients who received self-injected DMTs discontinued significantly sooner than fingolimod users. ${ }^{20}$

Oral DMTs offer advantages over injectable DMTs, such as convenience and ease of administration; however, it is less clear about factors that may affect adherence and persistence among oral DMTs. The efficacy of oral DMTs has not been compared in head-to-head trials, although all 3 of the oral DMTs in this study have demonstrated efficacy compared with placebo in clinical trials and are generally safe and well tolerated. ${ }^{27}$ 
Because each oral DMT in this study has a different mechanism of action, it is possible that patients may experience different levels of efficacy or tolerability that may affect treatment adherence or persistence.

Although there have been no direct head-to-head studies, indirect comparisons of the oral DMTs have been conducted. A recent Cochrane network meta-analytic review based on randomized controlled studies compared the benefit of 15 pharmacological treatments for RRMS and provided a ranking of these treatments. ${ }^{28}$ The network meta-analysis showed that fingolimod protected against the recurrence of relapse over 12 and 24 months, compared with placebo, more effectively than either dimethyl fumarate or teriflunomide.

Another indirect treatment comparison study showed that fingolimod had better outcomes (measured by the proportion of patients free from relapses and 3-month confirmed disability progression [clinical composite], free from gadoliniumenhancing T1 lesions and new or newly enlarged T2 lesions [magnetic resonance imaging composite], and free from all disease measures [overall composite]) compared with dimethyl fumarate or teriflunomide when tested against their respective trial populations. ${ }^{29}$

The indirect comparison studies conducted to date suggest that there remains a need to understand treatment outcome differences in direct comparison studies and to understand how those outcomes (e.g., clinical effectiveness and tolerability) may influence treatment adherence and persistence. Reasons for discontinuation or nonadherence were not assessed in our study; however, given the importance of high adherence and long-term use of DMTs in controlling relapses and reducing MS-related costs, ${ }^{18,19}$ this is an important area for future research.

\section{Limitations}

Some study limitations should be noted. The use of administrative claims in a retrospective study is associated with inherent limitations, including lack of data on patients' clinical presentations, the severity of disease, reasons for treatment discontinuation, and comprehensive health information. Adherence measured by administrative claims data may be imprecise. Further, adherence rates may not reflect actual adherence, since there is no way to ensure that patients took their medications as prescribed.

From administrative claims data, it is unknown which, if any, monitoring programs or manufacturer patient services (i.e., adherence-related programs) that dimethyl fumarate and teriflunomide patients may have participated in as they initiated treatment with their index therapies. Patients who start treatment with fingolimod must participate in a first-dose observation (FDO) program to monitor heart rate and blood pressure for a minimum of 6 hours. Patients who are required to go through this onboarding may have increased motivation to take medications as prescribed; however, it is uncertain how participation in the FDO process may have influenced adherence and persistence rates in this study.

The results of this study are generalizable to a U.S. commercially insured population of MS patients who were newly starting on oral DMTs and met study inclusion and exclusion criteria and may not reflect outcomes outside of this studied population. Also, the results may only be generalizable to the period used in the analysis, which was selected to reflect that dimethyl fumarate was the third oral DMT to enter the U.S. market in 2013.

In this study, we examined patients who were new initiators to the index DMT, and the study's identification period coincided with the market availability of dimethyl fumarate. Patients who were already using fingolimod and teriflunomide were not eligible for the study. We should note that the different sample sizes in each of our treatment cohorts was likely influenced by the timing of each drug's approval and availability in the U.S. market and the specific design of our study. As new DMTs enter the treatment landscape and more patients initiate treatment on these oral therapies, there may be a shift in treatment patterns seen in real-world settings.

\section{Conclusions}

In a real-world setting, MS patients who were newly prescribed fingolimod had significantly better adherence and persistence compared with patients newly treated with either dimethyl fumarate or teriflunomide over 12 months of follow-up from DMT initiation. Increased medication adherence and persistence are important factors when determining coverage decisions for MS patients to account for clinical and economic benefits. ${ }^{19}$

\section{Authors}

KRISTEN M. JOHNSON, PhD, MPH; FENG LIN, PhD, MS; JOHN J. KO, PharmD, MS; and VIVIAN HERRERA, DDS, MIA, $\mathrm{MPH}$, Novartis Pharmaceuticals, East Hanover, New Jersey. HUANXUE ZHOU, MS, KMK Consulting, Morristown, New Jersey.

AUTHOR CORRESPONDENCE: Kristen Johnson, PhD, MPH, 1 Health Plaza, East Hanover, NJ 07936. Tel.: 862.778.0882;

E-mail: Kristen_m.johnson@novartis.com.

\section{DISCLOSURES}

This research was funded by Novartis Pharmaceuticals. Johnson, Lin, Ko, and Herrera are employed by Novartis Pharmaceuticals and own Novartis stock. Huanxue Zhou is employed by KMK Consulting, which provides consulting services to Novartis.

Study concept and design were contributed by Johnson, Lin, Ko, and Herrera. Zhou collected the data, and data interpretation was performed by Johnson, Lin, Ko, and Herrera. All authors were involved in manuscript revision.

The abstract for this study was presented at the AMCP Nexus 2015; October 26-29, 2015; Orlando, Florida. 


\section{ACKNOWLEDGMENTS}

Editorial support for the preparation of this manuscript was provided by Ann Cameron, PhD, who is employed by Xcenda, and funded by Novartis Pharmaceuticals.

\section{REFERENCES}

1. National Multiple Sclerosis Society. What is MS? Available at: http://www. nationalmssociety.org/What-is-MS. Accessed June 24, 2017.

2. Naci H, Fleurence R, Birt J, Duhig A. Economic burden of multiple sclerosis: a systematic review of the literature. Pharmacoeconomics. 2010;28(5):363-79.

3. Tullman MJ. Overview of the epidemiology, diagnosis, and disease progression associated with multiple sclerosis. Am J Manag Care. 2013;19(2):S15-20.

4. Goodin DS. The epidemiology of multiple sclerosis: insights to disease pathogenesis. Handb Clin Neurol. 2014;(122):231-66.

5. National Multiple Sclerosis Society. Disease-modifying therapies for MS. Updated May 2017. Available at: http://www.nationalmssociety.org/ NationalMSSociety/media/MSNationalFiles/Brochures/Brochure-The-MSDisease-Modifying-Medications.pdf. Accessed June 24, 2017.

6. Gilenya (fingolimod) capsules, for oral use. Novartis Pharmaceuticals Corporation. Revised February 2016. Available at: https://www.pharma. us.novartis.com/sites/www.pharma.us.novartis.com/files/gilenya.pdf. Accessed June 24, 2017.

7. Aubagio (teriflunomide) tablets, for oral use. Genzyme Corporation Revised November 2016. Available at: http://products.sanofi.us/aubagio/ aubagio.pdf. Accessed June 24, 2017.

8. Tecfidera (dimethyl fumarate) delayed-release capsules, for oral use. Biogen Inc. Revised January 2017. Available at: https://www.tecfidera.com/ content/dam/commercial/multiple-sclerosis/tecfidera/pat/en_us/pdf/fullprescribing-info.pdf. Accessed June 24, 2017.

9. Multiple Sclerosis Coalition. The use of disease-modifying therapies in multiple sclerosis: principles and current evidence. A consensus paper. Updated March 2017. Available at: http://www.nationalmssociety.org/ NationalMSSociety/media/MSNationalFiles/Brochures/DMT_Consensus_ MS_Coalition.pdf. Accessed June 24, 2017.

10. Osterberg L, Blaschke T. Adherence to medication. New Eng J Med. 2005;353(5):487-97.

11. Cramer JA, Roy A, Burrell A, et al. Medication compliance and persistence: terminology and definitions. Value Health. 2008;11(1):44-47.

12. Menzin J, Caon C, Nichols C, White LA, Friedman M, Pill MW. Narrative review of the literature on adherence to disease-modifying therapies among patients with multiple sclerosis. J Manag Care Pharm. 2013;19(1 Suppl A):S24-40. Available at: http://www.jmcp.org/doi/10.18553/ jmcp.2013.19.sl.S24.

13. Reynolds MW, Stephen R, Seaman C, Rajagopalan K. Persistence and adherence to disease modifying drugs among patients with multiple sclerosis. Curr Med Res Opin. 2010;26(3):663-74.

14. Patti F. Optimizing the benefit of multiple sclerosis therapy: the importance of treatment adherence. Patient Prefer Adherence. 2010;4:1-9. Available at: http://www.ncbi.nlm.nih.gov/pmc/articles/PMC2819898/. Accessed June 24, 2017.
15. Bandari DS, Sternaman D, Chan T, Prostko CR, Sapir T. Evaluating risks, costs, and benefits of new and emerging therapies to optimize outcomes in multiple sclerosis. J Manag Care Pharm. 2012;18(9 Suppl B):1-17. Available at: http://www.jmcp.org/doi/10.18553/jmcp.2012.18.S9-B.1.

16. Starner CI, Alexander GC, Bowen K, Qiu Y, Wickersham PJ, Gleason PP. Specialty drug coupons lower out-of-pocket costs and may improve adherence at the risk of increasing premiums. Health Aff (Millwood). 2014;33(10):1761-69.

17. Wicks P, Brandes D, Park J, Liakhovitski D, Koudinova T, Sasane R. Preferred features of oral treatments and predictors of non-adherence: two web-based choice experiments in multiple sclerosis patients. Interact J Med Res. 2015;4(1):e6.

18. Ivanova JI, Bergman RE, Birnbaum HG, Phillips AL, Stewart M, Meletiche DM. Impact of medication adherence to disease-modifying drugs on severe relapse, and direct and indirect costs among employees with multiple sclerosis in the U.S. J Med Econ. 2012;15(3):601-09.

19. Tan H, Cai Q, Agarwal S, Stephenson JJ, Kamat S. Impact of adherence to disease-modifying therapies on clinical and economic outcomes among patients with multiple sclerosis. Adv Ther. 2011;28(1):51-61.

20. Agashivala N, Wu N, Abouzaid S, et al. Compliance to fingolimod and other disease modifying treatments in multiple sclerosis patients, a retrospective cohort study. BMC Neurol. 2013;13:138.

21. Bergvall N, Petrilla AA, Karkare SU, et al. Persistence with and adherence to fingolimod compared with other disease-modifying therapies for the treatment of multiple sclerosis: a retrospective U.S. claims database analysis. J Med Econ. 2014;17(10):696-707.

22. Chastek BJ, Oleen-Burkey M, Lopez-Bresnahan MV. Medical chart validation of an algorithm for identifying multiple sclerosis relapse in healthcare claims. J Med Econ. 2010;13(4):618-25.

23. Charlson ME, Pompei P, Ales KL, MacKenzie CR. A new method of classifying prognostic comorbidity in longitudinal studies: development and validation. J Chronic Dis. 1987;40(5):373-83.

24. Elixhauser A, Steiner C, Harris DR, Coffey RM. Comorbidity measures for use with administrative data. Med Care. 1998;36(1):8-27.

25. Quan H, Sundararajan V, Halfon P, et al. Coding algorithms for defining comorbidities in ICD-9-CM and ICD-10 administrative data. Med Care. 2005;43(11):1130-39.

26. Andrade SE, Kahler KH, Frech F, Chan KA. Methods for evaluation of medication adherence and persistence using automated databases. Pharmacoepidemiol Drug Saf. 2006;15(8):565-74.

27. Weinstock-Guttman B. An update on new and emerging therapies for relapsing-remitting multiple sclerosis. Am J Manag Care. 2013;19 (17 Suppl):S343-54.

28. Tramacere I, DelGiovane C, Salanti G, D’Amico R, Filippini G Immunomodulators and immunosuppressants for relapsing remitting multiple sclerosis: a network meta-analysis. Cochr Datab Syst Rev. 2015;9:CD011381

29. Nixon R, Bergvall N, Tomic D, Sfikas N, Cutter G, Giovannoni G. No evidence of disease activity: indirect comparisons of oral therapies for the treatment of relapsing-remitting multiple sclerosis. Adv Ther. 2014:31(11):1134-54. 